\title{
On Fractional Integro-differential Equations with State-Dependent Delay and Non-Instantaneous Impulses
}

\author{
Khalida Aissani $^{1}$, Mouffak Benchohra 211 and Nadia Benkhettou ${ }^{2}$ \\ 1 Laboratory of Mathematics, Djillali Liabes University of Sidi Bel-Abbès, \\ PO Box 89, 22000, Sidi Bel-Abbès, Algeria. \\ 2 University of Bechar \\ PO Box 417, 08000, Bechar, Algeria \\ benchohra@yahoo.com, aissani_k@yahoo.fr
}

\begin{abstract}
In this paper, we prove the existence of mild solution of the fractional integro-differential equations with state-dependent delay with not instantaneous impulses. The existence results are obtained under the conditions in respect of Kuratowski's measure of noncompactness. An example is also given to illustrate the results.
\end{abstract}

\section{RESUMEN}

En este artículo, demostramos la existencia de soluciones mild de ecuaciones integrodiferenciales fraccionarias con retardo dependiente del estado e impulsos no instantáneos. Los resultados de existencia se obtienen bajo condiciones respecto de la medida de Kuratowski de no compacidad. También se entrega un ejemplo para ilustrar los resultados.

Keywords and Phrases: Non-instantaneous impulsive conditions, fractional integro-differential equations, Caputo fractional derivative, mild solution, fixed point, state-dependent delay.

2010 AMS Mathematics Subject Classification: 26A33, 34A12, 34A37, 34G20.

${ }^{1}$ Corresponding author 


\section{Introduction}

Fractional differential equations play the crucial and significant role in the field of science and engineering. Most importantly non-integer order differential equations have ability to describe the real behavior and memory effects of the system and processes. For more details about fractional differential equations and its applications refer the monographs of Abbas et al. [1, 2, 3], Baleanu et al. [12, Diethelm [18, Hilfer [24], Kilbas et al. [26], Miller and Ross [32], Samko et al. 37], Tarasov 38, and Zhou 39] and the references therein.

Most of the research papers deal with the existence of solutions for differential equations with instantaneous impulsive conditions see [6, 7, 10, 11, 14, 28, 31. But many times it has seen that certain dynamics of evolution processes cannot describe by instantaneous impulses, For instance: Pharmacotherapy, high or low levels of glucose, this situation can be interpreted as an impulsive action which starts abruptly at certain point of time and continue with a finite time interval. Such type of systems are known as non-instantaneous impulsive systems which are more suitable to study the dynamics of evolution processes [4].

This theory of a new class of impulsive differential equation was initiated by Hernández et al. [23]. Afterwards, Pierri et al. 35] continued the work in this field and extend the theory of [23] in a $\mathrm{PC}_{\alpha}$ normed Banach space. The existence of solutions for non-instantaneous impulsive fractional differential equations have also been discussed in [8, 19, 27, 29, 34].

Recently, Benchohra et al. [15] investigated the existence and uniqueness of solutions on a compact interval for non-linear fractional integro-differential equations with state-dependent delay and noninstantaneous impulses. Anguraj and Kanjanadevi 9] studied the existence and uniqueness of fractional neutral differential equations with state-dependent delay subject to non-instantaneous impulsive conditions.

Motivated by the papers cited above, in this paper, we consider the existence of mild solutions for fractional integro-differential equations with state-dependent delay and non instantaneous impulses described by the form

$$
\begin{aligned}
& { }^{c} D_{t}^{q} x(t)+A x(t)=\int_{0}^{t} a(t, s) f\left(s, x_{\rho\left(s, x_{s}\right)}, x(s)\right) d s, \text { a.e. } t \in\left(s_{i}, t_{i+1}\right] \subset J, i=0, \ldots, N, \\
& x(t)=h_{i}\left(t, x_{\rho\left(t, x_{t}\right)}, x(t)\right), \quad t \in\left(t_{i}, s_{i}\right], i=1, \ldots, N, \\
& x_{0}=\phi \in \mathcal{B} \text {, }
\end{aligned}
$$

where ${ }^{C} D_{t}^{q}$ is the Caputo fractional derivative of order $0<q<1, A: D(A) \subset X \rightarrow X$ is the infinitesimal generator of an analytic semigroup $\{S(t)\}_{t \geq 0}$ of uniformly bounded linear operators on $X, f: J \times \mathcal{B} \times X \longrightarrow X, J=[0, T], T>0$, and $\rho: J \times \mathcal{B} \rightarrow(-\infty, T]$ are appropriate functions, $a: D \rightarrow \mathbb{R}(D=\{(t, s) \in J \times J: t \geq s\})$. Here $0=t_{0}=s_{0}<t_{1} \leq s_{1} \leq t_{2}<\ldots<t_{N-1} \leq s_{N} \leq$ $t_{N} \leq t_{N+1}=T$ are pre-fixed numbers, and $h_{i} \in C\left(\left(t_{i}, s_{i}\right] \times \mathcal{B} \times X, X\right)$, for all $i=1,2, \ldots, N$. For 
any continuous function $x$ defined on $(-\infty, T]$ and any $t \in J$, we denote by $x_{t}$ the element of $\mathcal{B}$ defined by

$$
x_{t}(\theta)=x(t+\theta), \quad \theta \in(-\infty, 0] .
$$

Here $x_{t}$ represents the history of the state up to the present time $t$ and $\phi \in \mathcal{B}$ to be specified later.

\section{Preliminaries}

Let $(X,\|\cdot\|)$ be a real Banach space.

$\mathrm{C}=\mathrm{C}(\mathrm{J}, \mathrm{X})$ be the space of all $\mathrm{X}$-valued continuous functions on $\mathrm{J}$.

$L(X)$ be the Banach space of all linear and bounded operators on $X$.

$L^{1}(J, X)$ the space of $X$-valued Bochner integrable functions on $J$ with the norm

$$
\|y\|_{L^{1}}=\int_{0}^{T}\|y(t)\| d t
$$

$L^{\infty}(J, \mathbb{R})$ is the Banach space of measurable functions which are essentially bounded, normed by

$$
\|y\|_{L_{\infty}}=\inf \{d>0:|y(t)| \leq d, \text { a.e. } t \in J\} .
$$

We need some basic definitions of the fractional calculus theory which are used in this paper.

Definition 2.1. Let $\alpha>0$ and $\mathrm{f}: \mathbb{R}_{+} \rightarrow \mathrm{X}$ be in $\mathrm{L}^{1}\left(\mathbb{R}_{+}, \mathrm{X}\right)$. Then the Riemann-Liouville integral is given by:

$$
\mathrm{I}_{\mathrm{t}}^{\alpha} \mathrm{f}(\mathrm{t})=\frac{1}{\Gamma(\alpha)} \int_{0}^{\mathrm{t}} \frac{\mathrm{f}(\mathrm{s})}{(\mathrm{t}-\mathrm{s})^{1-\alpha}} \mathrm{ds}
$$

where $\Gamma(\cdot)$ is the Euler gamma function.

For more details on the Riemann-Liouville fractional derivative, we refer the reader to [17.

Definition 2.2. [36] The Caputo derivative of order $\alpha$ for a function $\mathrm{f}:[0,+\infty) \rightarrow \mathrm{X}$ can be written as

$$
D_{t}^{\alpha} f(t)=\frac{1}{\Gamma(n-\alpha)} \int_{0}^{t} \frac{f^{(n)}(s)}{(t-s)^{\alpha+1-n}} d s=I^{n-\alpha} f^{(n)}(t), \quad t>0, n-1 \leq \alpha<n .
$$

If $0 \leq \alpha<1$, then

$$
D_{t}^{\alpha} f(t)=\frac{1}{\Gamma(1-\alpha)} \int_{0}^{t} \frac{f^{(1)}(s)}{(t-s)^{\alpha}} d s
$$

Obviously, The Caputo derivative of a constant is equal to zero.

Definition 2.3. A function $\mathrm{f}: \mathrm{J} \times \mathcal{B} \times \mathrm{X} \longrightarrow \mathrm{X}$ is said to be an Carathéodory function if it satisfies

(i) for each $\mathrm{t} \in \mathrm{J}$ the function $\mathrm{f}(\mathrm{t}, \cdot, \cdot): \mathcal{B} \times \mathrm{X} \longrightarrow \mathrm{X}$ is continuous; 
(ii) for each $(v, w) \in \mathcal{B} \times \mathrm{X}$ the function $\mathrm{f}(\cdot, v, w): \mathrm{J} \rightarrow \mathrm{X}$ is measurable.

Next we give the concept of a measure of noncompactness [13.

Definition 2.4. Let B be a bounded subset of a Banach space Y. The Kuratowski measure of noncompactness of $\mathrm{B}$ is defined as

$$
\alpha(B)=\inf \{\mathrm{d}>0: \mathrm{B} \text { has a finite cover by sets of diameter } \leq \mathrm{d}\} .
$$

We note that this measure of noncompactness satisfies the properties ([13]).

\section{Lemma 2.5.}

1. If $\mathrm{A} \subseteq \mathrm{B}$ then $\alpha(\mathrm{A}) \leq \alpha(\mathrm{B})$,

2. $\alpha(A)=\alpha(\bar{A})$, where $\bar{A}$ denotes the closure of $A$,

3. $\alpha(A)=0 \Leftrightarrow \bar{A}$ is compact (A is relatively compact),

4. $\alpha(\lambda A)=|\lambda| A$, with $\lambda \in \mathbb{R}$,

5. $\alpha(A \cup B)=\max \{\alpha(A), \alpha(B)\}$,

6. $\alpha(A+B) \leq \alpha(A)+\alpha(B)$, where

$$
A+B=\{x+y: x \in A, y \in B\}
$$

7. $\alpha(A+a)=\alpha(A)$ for any $a \in X$,

8. $\alpha(\overline{\operatorname{conv}} A)=\alpha(A)$, where $\overline{\operatorname{conv}} A$ is the closed convex hull of $A$.

For $\mathrm{H} \subset \mathrm{C}(\mathrm{J}, \mathrm{X})$, we define

$$
\int_{0}^{t} H(s) d s=\left\{\int_{0}^{t} u(s) d s: u \in H\right\} \quad \text { for } t \in J,
$$

where $H(s)=\{u(s) \in X: u \in H\}$.

Lemma 2.6. [13] If $\mathrm{H} \subset \mathrm{C}(\mathrm{J}, \mathrm{X})$ is a bounded, equicontinuous set, then

$$
\alpha_{C}(H)=\sup _{t \in J} \alpha(H(t)) .
$$

Lemma 2.7. [21] If $\left\{\mathrm{u}_{\mathrm{n}}\right\}_{\mathrm{n}=1}^{\infty} \subset \mathrm{L}^{1}(\mathrm{~J}, \mathrm{X})$ and there exists $\mathrm{m} \in \mathrm{L}^{1}\left(\mathrm{~J}, \mathbb{R}^{+}\right)$such that $\left\|\mathrm{u}_{\mathrm{n}}(\mathrm{t})\right\| \leq \mathrm{m}(\mathrm{t})$, a.e. $\mathrm{t} \in \mathrm{J}$, then $\alpha\left(\left\{\mathrm{u}_{\mathrm{n}}(\mathrm{t})\right\}_{\mathrm{n}=1}^{\infty}\right)$ is integrable and

$$
\alpha\left(\left\{\int_{0}^{t} u_{n}(s) d s\right\}_{n=1}^{\infty}\right) \leq 2 \int_{0}^{t} \alpha\left(\left\{u_{n}(s)\right\}_{n=1}^{\infty}\right) d s .
$$


In this paper, we will employ an axiomatic definition for the phase space $\mathcal{B}$ which is similar to those introduced by Hale and Kato $[20$. Specifically, $\mathcal{B}$ will be a linear space of functions mapping $(-\infty, 0]$ into $X$ endowed with a seminorm $\|\cdot\|_{\mathcal{B}}$, and satisfies the following axioms:

(A1) If $x:(-\infty, T] \longrightarrow X$ is continuous on $J$ and $x_{0} \in \mathcal{B}$, then $x_{t} \in \mathcal{B}$ and $x_{t}$ is continuous in $t \in J$ and

$$
\|x(t)\| \leq \mathrm{C}\left\|x_{\mathrm{t}}\right\|_{\mathcal{B}}
$$

where $\mathrm{C} \geq 0$ is a constant.

(A2) There exist a continuous function $C_{1}(t)>0$ and a locally bounded function $C_{2}(t) \geq 0$ in $t \geq 0$ such that

$$
\left\|x_{t}\right\|_{\mathcal{B}} \leq C_{1}(t) \sup _{s \in[0, t]}\|x(s)\|+C_{2}(t)\left\|x_{0}\right\|_{\mathcal{B}}
$$

for $\mathrm{t} \in[0, \mathrm{~T}]$ and $\mathrm{x}$ as in $(\mathrm{A} 1)$.

(A3) The space $\mathcal{B}$ is complete.

Remark 2.8. Condition 2.4) in (A1) is equivalent to $\|\phi(0)\| \leq \mathrm{C}\|\phi\|_{\mathcal{B}}$, for all $\phi \in \mathcal{B}$.

Example 2.9. The phase space $\mathrm{C}_{\mathrm{r}} \times \mathrm{L}^{\mathrm{p}}(\mathrm{g}, \mathrm{X})$.

Let $\mathrm{r} \geq 0,1 \leq \mathrm{p}<\infty$, and let $\mathrm{g}:(-\infty,-\mathrm{r}) \rightarrow \mathbb{R}$ be a nonnegative measurable function which satisfies the conditions $(\mathrm{g}-5),(\mathrm{g}-6)$ in the terminology of [25]. Briefly, this means that $\mathrm{g}$ is locally integrable and there exists a nonnegative, locally bounded function $\Lambda$ on $(-\infty, 0]$, such that $g(\xi+\theta) \leq \Lambda(\xi) g(\theta)$, for all $\xi \leq 0$ and $\theta \in(-\infty,-r) \backslash N_{\xi}$, where $N_{\xi} \subseteq(-\infty,-r)$ is a set with Lebesgue measure zero.

The space $\mathrm{C}_{\mathrm{r}} \times \mathrm{L}^{\mathrm{p}}(\mathrm{g}, \mathrm{X})$ consists of all classes of functions $\varphi:(-\infty, 0] \rightarrow \mathrm{X}$, such that $\varphi$ is continuous on $[-\mathrm{r}, 0]$, Lebesgue-measurable, and $\mathrm{g}\|\varphi\|^{\mathrm{p}}$ on $(-\infty,-\mathrm{r})$. The seminorm in $\|\cdot\|_{\mathcal{B}}$ is defined by

$$
\|\varphi\|_{\mathcal{B}}=\sup _{\theta \in[-r, 0]}\|\varphi(\theta)\|+\left(\int_{-\infty}^{-r} g(\theta)\|\varphi(\theta)\|^{p} \mathrm{~d} \theta\right)^{\frac{1}{p}} .
$$

The space $\mathcal{B}=\mathrm{C}_{\mathrm{r}} \times \mathrm{L}^{\mathrm{p}}(\mathrm{g}, \mathrm{X})$ satisfies axioms (A1), (A2), (A3). Moreover, for $\mathrm{r}=0$ and $\mathrm{p}=2$, this space coincides with $\mathrm{C}_{0} \times \mathrm{L}^{2}(\mathrm{~g}, \mathrm{X}), \mathrm{H}=1, \mathrm{M}(\mathrm{t})=\Lambda(-\mathrm{t})^{\frac{1}{2}}, \mathrm{~K}(\mathrm{t})=1+\left(\int_{-\mathrm{r}}^{0} \mathrm{~g}(\tau) \mathrm{d} \tau\right)^{\frac{1}{2}}$, for $\mathrm{t} \geq 0$ (see [25], Theorem 1.3.8 for details).

For our purpose we will only need the following fixed point theorems.

Theorem 2.10. [5, 33] Let $\mathrm{U}$ be a bounded, closed and convex subset of a Banach space, and let $\mathrm{N}$ be a continuous mapping of $\mathrm{U}$ into itself. If the implication

$$
\mathrm{V}=\overline{\operatorname{conv}} \mathrm{N}(\mathrm{V}) \text { or } \mathrm{V}=\mathrm{N}(\mathrm{V}) \cup\{0\} \Longrightarrow \alpha(\mathrm{V})=0
$$

holds for every subset $\mathrm{V}$ of $\mathrm{U}$, then $\mathrm{N}$ has a fixed point. 
A continuous map $\mathrm{N}: \mathrm{D} \subseteq \mathrm{E} \rightarrow \mathrm{E}$ is said to be a $\alpha$-contraction if there exists a constant $v \in[0,1)$ such that $\alpha(\mathrm{N}(\mathrm{C})) \leq v \alpha(\mathrm{C})$ for any bounded closed subset $\mathrm{C} \subseteq \mathrm{D}$.

Theorem 2.11. (Darbo-Sadovskii) [13] Let $\mathrm{E}$ be a Banach space. If $\mathrm{D} \subseteq \mathrm{E}$ is bounded closed and convex, the continuous map $\mathrm{N}: \mathrm{D} \rightarrow \mathrm{D}$ is a $\alpha$-contraction, then the map $\mathrm{N}$ has at least one fixed point in D.

Consider the space

$$
P C(J, X)=\left\{x: J \rightarrow X, x \in C\left(J \cap\left(\cup_{k=0}^{N}\left(t_{k}, s_{k}\right]\right), X\right),\right.
$$

and $x\left(t_{\mathrm{k}}^{+}\right), x\left(s_{\mathrm{k}}^{-}\right)$exist with, $\left.x\left(s_{\mathrm{k}}^{-}\right)=x\left(s_{\mathrm{k}}\right), \mathrm{k}=1, \ldots, \mathrm{N}\right\}$.

Obviously, $\mathrm{PC}(\mathrm{J}, \mathrm{X})$ is a Banach space with the norm

$$
\|x\|_{P C}=\sup _{t \in J}\|x(t)\|
$$

\section{$3 \quad$ Existence Results}

In this section, we prove the existence of mild solution of (1.1).

Definition 3.1. A function $\mathrm{x}:(-\infty, \mathrm{T}] \rightarrow \mathrm{X}$ is said to be a mild solution of the equation (1.1) if $\mathrm{x}_{0}=\phi$ on $(-\infty, \mathrm{T}],\left.\mathrm{x}\right|_{[0, \mathrm{~T}]} \in \mathrm{PC}([0, \mathrm{~T}], \mathrm{X})$ and $\mathrm{x}$ satisfies

$$
x(t)= \begin{cases}Q(t) \phi(0)+\int_{0}^{t} \int_{0}^{s} R(t-s) a(s, \tau) f\left(\tau, x_{\rho\left(\tau, x_{\tau}\right)}, x(\tau)\right) d \tau d s, & t \in\left[0, t_{1}\right], \\ h_{i}\left(t, x_{\rho\left(t, x_{t}\right)}, x(t)\right), & t \in\left(t_{i}, s_{i}\right], i=1,2, \ldots, N, \\ Q\left(t-s_{i}\right) h_{i}\left(s_{i}, x_{\rho\left(s_{i}, x_{s_{i}}\right)}, x\left(s_{i}\right)\right) & \\ +\int_{0}^{t} \int_{0}^{s} R(t-s) a(s, \tau) f\left(\tau, x_{\rho\left(\tau, x_{\tau}\right)}, x(\tau)\right) d \tau d s, & t \in\left(s_{i}, t_{i+1}\right],\end{cases}
$$

where

$$
\mathrm{Q}(\mathrm{t})=\int_{0}^{\infty} \xi_{\mathrm{q}}(\sigma) S\left(\mathrm{t}^{\mathrm{q}} \sigma\right) \mathrm{d} \sigma, \quad \mathrm{R}(\mathrm{t})=\mathrm{q} \int_{0}^{\infty} \sigma \mathrm{t}^{\mathrm{q}-1} \xi_{\mathrm{q}}(\sigma) S\left(\mathrm{t}^{\mathrm{q}} \sigma\right) d \sigma
$$

and $\xi_{\mathrm{q}}$ is a probability density function defined on $(0, \infty)$ such that

$$
\xi_{q}(\sigma)=\frac{1}{q} \sigma^{-1-\left(\frac{1}{q}\right)} \varpi_{q}\left(\sigma^{-\frac{1}{q}}\right) \geq 0,
$$


where

$$
\varpi_{\mathrm{q}}(\sigma)=\frac{1}{\pi} \sum_{\mathrm{k}=1}^{\infty}(-1)^{\mathrm{k}-1} \sigma^{-\mathrm{q} k-1} \frac{\Gamma(\mathrm{kq}+1)}{\mathrm{k} !} \sin (\mathrm{k} \pi \mathrm{q}), \quad \sigma \in(0, \infty) .
$$

Remark 3.2. Note that $\{\mathrm{S}(\mathrm{t})\}_{\mathrm{t} \geq 0}$ is a uniformly bounded i.e

$$
\text { there exists a constant } M>0 \text { such that }\|S(t)\|_{L(X)} \leq M \text { for all } t \geq 0 \text {. }
$$

Remark 3.3. According to [30], direct calculation gives that

$$
\|R(t)\| \leq C_{q, M} t^{q-1}, \quad t>0
$$

where $\mathrm{C}_{\mathrm{q}, \mathrm{M}}=\frac{\mathrm{qM}}{\Gamma(1+\mathrm{q})}$.

Set

$$
\mathcal{R}\left(\rho^{-}\right)=\{\rho(s, \varphi):(s, \varphi) \in \mathrm{J} \times \mathcal{B}, \rho(s, \varphi) \leq 0\} .
$$

We always assume that $\rho: \mathrm{J} \times \mathcal{B} \rightarrow(-\infty, \mathrm{T}]$ is continuous. Additionally, we introduce following hypothesis:

$\left(\mathrm{H}_{\varphi}\right)$ The function $t \rightarrow \varphi_{\mathrm{t}}$ is continuous from $\mathcal{R}\left(\rho^{-}\right)$into $\mathcal{B}$ and there exists a continuous and bounded function $\mathrm{L}^{\phi}: \mathcal{R}\left(\rho^{-}\right) \rightarrow(0, \infty)$ such that

$$
\left\|\phi_{\mathrm{t}}\right\|_{\mathcal{B}} \leq \mathrm{L}^{\Phi}(\mathrm{t})\|\phi\|_{\mathcal{B}} \quad \text { for every } \mathrm{t} \in \mathcal{R}\left(\rho^{-}\right) .
$$

Remark 3.4. Condition $\left(\mathrm{H}_{\varphi}\right)$, is frequently verified by the continuous and bounded functions. For more details see [25].

Remark 3.5. In the rest of this section, $\mathrm{C}_{1}^{*}$ and $\mathrm{C}_{2}^{*}$ are the constants

$$
C_{1}^{*}=\sup _{s \in J} C_{1}(s) \text { and } C_{2}^{*}=\sup _{s \in J} C_{2}(s) .
$$

Lemma 3.6. [22] If $x: \mathbb{R} \rightarrow X$ is a function such that $x_{0}=\phi$, then

$$
\left\|x_{s}\right\|_{\mathcal{B}} \leq\left(C_{2}^{*}+L^{\phi}\right)\|\phi\|_{\mathcal{B}}+C_{1}^{*} \sup \{|x(\theta)| ; \theta \in[0, \max \{0, s\}]\}, s \in \mathcal{R}\left(\rho^{-}\right) \cup \mathrm{J},
$$

where $\mathrm{L}^{\phi}=\sup _{\mathrm{t} \in \mathcal{R}\left(\rho^{-}\right)} \mathrm{L}^{\phi}(\mathrm{t})$.

Let us introduce the following hypotheses:

(H1) $\mathrm{f}: \mathrm{J} \times \mathcal{B} \times \mathrm{X} \longrightarrow \mathrm{X}$ satisfies the Carathéodory conditions.

(H2) There exist functions $\mu, \mu^{*} \in \mathrm{L}^{1}\left(\mathrm{~J}, \mathbb{R}^{+}\right)$and continuous nondecreasing functions $\psi, \psi^{*}: \mathbb{R}^{+} \rightarrow$ $(0,+\infty)$ such that

$$
\begin{gathered}
\|f(t, x, y)\| \leq \mu(t) \psi\left(\|x\|_{\mathcal{B}}+\|y\|\right), \quad(t, x, y) \in J \times \mathcal{B} \times X, \\
\left\|h_{i}(t, x, y)\right\| \leq \mu^{*}(t) \psi^{*}\left(\|x\|_{\mathcal{B}}+\|y\|\right), \quad(t, x, y) \in J \times \mathcal{B} \times X,
\end{gathered}
$$


(H3) For any bounded sets $\mathrm{D}_{1} \subset \mathcal{B}, \mathrm{D}_{2} \subset \mathrm{X}$, and $0 \leq \mathrm{s} \leq \mathrm{t} \leq \mathrm{T}$, there exists an integrable positive function $\eta$ such that

$$
\alpha\left(R(t-s) f\left(\tau, D_{1}, D_{2}\right)\right) \leq \eta_{t}(s, \tau)\left(\alpha\left(D_{2}\right)+\sup _{-\infty<\theta \leq 0} \alpha\left(D_{1}(\theta)\right)\right)
$$

where $\eta_{t}(s, \tau)=\eta(t, s, \tau)$ and $\sup _{t \in J} \int_{0}^{t} \int_{0}^{s} \eta_{t}(s, \tau) d \tau d s=\eta^{*}<\infty$.

(H4) There exists a constant $\mathrm{L}>0$ such that, for each bounded sets $\mathrm{D}_{1} \subset \mathcal{B}, \mathrm{D}_{2} \subset \mathrm{X}$,

$$
\alpha\left(h_{i}\left(\tau, D_{1}, D_{2}\right)\right) \leq L\left(\alpha\left(D_{2}\right)+\sup _{-\infty<\theta \leq 0} \alpha\left(D_{1}(\theta)\right)\right)
$$

(H5) For each $t \in J, a(t, s)$ is measurable on $[0, t]$ and $a(t)=\operatorname{ess} \sup \{|a(t, s)|, 0 \leq s \leq t\}$ is bounded on $J$. The map $t \rightarrow a_{t}$ is continuous from $J$ to $L^{\infty}(J, \mathbb{R})$, here, $a_{t}(s)=a(t, s)$.

Set $a=\sup _{t \in J} a(t)$.

Our first result is based on the Mönch fixed point theorem.

Theorem 3.7. Suppose that the assumptions $\left(\mathrm{H}_{\varphi}\right),(\mathrm{H} 1)-(\mathrm{H} 5)$ hold, and if

$$
2 \mathrm{ML}+16 \text { a } \eta^{*}<1
$$

then the problem (1.1) has at least one mild solution.

Proof. Let $Y=\{u \in P C(X): u(0)=\phi(0)=0\}$ endowed with the uniform convergence topology and define the operator $\mathrm{P}: \mathrm{Y} \rightarrow \mathrm{Y}$ by

$$
P(x)(t)= \begin{cases}Q(t) \phi(0)+\int_{0}^{t} \int_{0}^{s} R(t-s) a(s, \tau) f\left(\tau, \bar{x}_{\rho\left(\tau, \bar{x}_{\tau}\right)}, \bar{x}(\tau)\right) d \tau d s, & t \in\left[0, t_{1}\right], \\ h_{i}\left(t, \bar{x}_{\rho\left(t, \bar{x}_{t}\right)}, \bar{x}(t)\right), & t \in\left(t_{i}, s_{i}\right], i=1,2, \ldots, N, \\ Q\left(t-s_{i}\right) h_{i}\left(s_{i}, \bar{x}_{\rho\left(s_{i}, \bar{x}_{s_{i}}\right)}, \bar{x}\left(s_{i}\right)\right) & \\ +\int_{s_{i}}^{t} \int_{0}^{s} R(t-s) a(s, \tau) f\left(\tau, \bar{x}_{\rho\left(\tau, \bar{x}_{\tau}\right)}, \bar{x}(\tau)\right) d \tau d s, & t \in\left(s_{i}, t_{i+1}\right],\end{cases}
$$

where $x:(-\infty, T] \rightarrow X$ is such that $x_{0}=\phi$ and $\bar{x}=x$ on $J$. Let $\bar{\phi}:(-\infty, T] \longrightarrow X$ be the extension of $\phi$ to $(-\infty, \mathrm{T}]$ such that $\bar{\phi}(\theta)=\phi(0)=0$ on $\mathrm{J}$.

Choose

$r \geq M\left\|\mu^{*}\right\|_{\mathrm{L}^{1}} \psi^{*}\left(\left(\mathrm{C}_{2}^{*}+\mathrm{L}^{\phi}\right)\|\phi\|_{\mathcal{B}}+\left(\mathrm{C}_{1}^{*}+1\right) r\right)+a \mathrm{C}_{\mathrm{q}, M}\|\mu\|_{\mathrm{L}^{1}} \frac{\mathrm{T}^{\mathrm{q}}}{\mathrm{q}} \psi\left(\left(\mathrm{C}_{2}^{*}+\mathrm{L}^{\phi}\right)\|\phi\|_{\mathcal{B}}+\left(\mathrm{C}_{1}^{*}+1\right) r\right)$ and define the set

$$
\mathrm{B}_{\mathrm{r}}=\left\{x \in \mathrm{Y}:\|x\|_{\mathrm{PC}} \leq \mathrm{r}\right\}
$$


then $B_{r}$ is a bounded, closed-convex subset in $Y$.

Step 1: $\mathrm{P}$ is continuous.

Let $\left\{x^{k}\right\}_{k \in \mathbb{N}}$ be a sequence such that $x^{k} \rightarrow x$ in $B_{r}$ as $k \rightarrow \infty$.

Case 1. For each $t \in\left[0, t_{1}\right]$, we have

$$
\begin{aligned}
\left\|P\left(x^{k}\right)(t)-P(x)(t)\right\| & \leq \int_{0}^{t} \int_{0}^{s}\|R(t-s)\|\|a(s, \tau)\| \| f\left(\tau, \bar{x}_{\rho\left(\tau, \bar{x}_{\tau}^{k}\right)}^{k}, \bar{x}^{k}(\tau)\right) \\
& -f\left(\tau, \bar{x}_{\rho\left(\tau, \bar{x}_{\tau}\right)}, \bar{x}(\tau)\right) \| d \tau d s \\
& \leq a C_{q, M} \int_{0}^{t} \int_{0}^{s}(t-s)^{q-1} \| f\left(\tau, \bar{x}_{\rho\left(\tau, \bar{x}_{\tau}^{k}\right)}^{k}, \bar{x}^{k}(\tau)\right) \\
& -f\left(\tau, \bar{x}_{\rho\left(\tau, \bar{x}_{\tau}\right)}, \bar{x}(\tau)\right) \| d \tau d s .
\end{aligned}
$$

Case 2. For each $t \in\left[t_{i}, s_{i}\right), i=1,2, \ldots, N$, we have

$$
\begin{aligned}
\left\|P\left(x^{k}\right)(t)-P(x)(t)\right\| & =\left\|h_{i}\left(t, \bar{x}_{\rho\left(t, \bar{x}_{t}^{k}\right)}^{k}, \bar{x}^{k}(t)\right)-h_{i}\left(t, \bar{x}_{\rho\left(t, \bar{x}_{t}\right)}, \bar{x}(t)\right)\right\| \\
& \rightarrow 0 \quad k \rightarrow \infty .
\end{aligned}
$$

Case 3. For each $t \in\left(s_{i}, t_{i+1}\right], i=1,2, \ldots, N$, we obtain

$$
\begin{aligned}
\left\|P\left(x^{k}\right)(t)-P(x)(t)\right\| & \leq\left\|Q\left(t-s_{i}\right)\right\|\left\|h_{i}\left(s_{i}, \bar{x}_{\rho\left(s_{i}, \bar{x}_{s_{i}}^{k}\right)}^{k}, \bar{x}^{k}\left(s_{i}\right)\right)-h_{i}\left(s_{i}, \bar{x}_{\rho\left(s_{i}, \bar{x}_{s_{i}}\right)}, \bar{x}\left(s_{i}\right)\right)\right\| \\
& +\int_{s_{i}}^{t} \int_{0}^{s}\|R(t-s)\|\|\mathrm{a}(s, \tau)\| \| f\left(\tau, \bar{x}_{\rho\left(\tau, \bar{x}_{\tau}^{k}\right)}^{k}, \bar{x}^{k}(\tau)\right) \\
& -\mathrm{f}\left(\tau, \bar{x}_{\rho\left(\tau, \bar{x}_{\tau}\right)}, \bar{x}(\tau)\right) \| \mathrm{d} \tau d s \\
& \leq M\left\|h_{i}\left(s_{i}, \bar{x}_{\rho\left(s_{i}, \bar{x}_{s_{i}}^{k}\right.}^{k}, \bar{x}^{k}\left(s_{i}\right)\right)-h_{i}\left(s_{i}, \bar{x}_{\rho\left(s_{i}, \bar{x}_{s_{i}}\right)}, \bar{x}\left(s_{i}\right)\right)\right\| \\
& +a C_{q, M} \int_{s_{i}}^{t} \int_{0}^{s}(t-s)^{q-1} \| f\left(\tau, \bar{x}_{\rho\left(\tau, \bar{x}_{\tau}^{k}\right)}^{k}, \bar{x}^{k}(\tau)\right) \\
& -f\left(\tau, \bar{x}_{\rho\left(\tau, \bar{x}_{\tau}\right)}, \bar{x}(\tau)\right) \| d \tau d s .
\end{aligned}
$$

Since the function $h_{i}$ is continuous and $f$ is of Carathéodory type, we have by the Lebesgue dominated convergence theorem that

$$
\left\|\mathrm{P}\left(\mathrm{x}^{\mathrm{k}}\right)(\mathrm{t})-\mathrm{P}(\mathrm{x})(\mathrm{t})\right\| \rightarrow 0 \quad \text { as } \mathrm{k} \rightarrow \infty
$$

which shows the operator $\mathrm{P}$ is continuous.

Step 2: $P$ maps $B_{r}$ into itself. 
Case 1. For all $t \in\left[0, t_{1}\right]$, we get

$$
\begin{aligned}
& \|P(x)(t)\| \leq\|Q(t) \phi(0)\|+\int_{0}^{t} \int_{0}^{s}\left\|R(t-s) a(s, \tau) f\left(\tau, \bar{x}_{\rho\left(\tau, \bar{x}_{\tau}\right)}, \bar{x}(\tau)\right)\right\| d \tau d s \\
& \leq M C\|\phi\|_{\mathcal{B}}+a C_{q, M} \int_{0}^{t} \int_{0}^{s}(t-s)^{q-1} \mu(\tau) \psi\left(\left\|\bar{x}_{\rho\left(\tau, \bar{x}_{\tau}\right)}\right\|_{\mathcal{B}}+\|\bar{x}\|\right) d \tau d s \\
& \leq M C\|\phi\|_{\mathcal{B}}+a C_{q, M} \int_{0}^{t} \int_{0}^{s}(t-s)^{q-1} \mu(\tau) \\
& \times \quad \psi\left(\left(C_{2}^{*}+L^{\phi}\right)\|\phi\|_{\mathcal{B}}+C_{1}^{*} r+r\right) d \tau d s \\
& \leq M C\|\phi\|_{\mathcal{B}}+a C_{q, M}\|\mu\|_{L^{1}} \frac{T^{q}}{q} \psi\left(\left(C_{2}^{*}+L^{\phi}\right)\|\phi\|_{\mathcal{B}}+\left(C_{1}^{*}+1\right) r\right) \\
& \leq \text { r. }
\end{aligned}
$$

Case 2. For all $t \in\left[t_{i}, s_{i}\right), i=1,2, \ldots, N$, we have

$$
\begin{aligned}
& \|\mathrm{P}(\mathrm{x})(\mathrm{t})\| \leq\left\|\mathrm{h}_{\mathrm{i}}\left(\mathrm{t}, \overline{\mathrm{x}}_{\rho\left(\mathrm{t}, \overline{\mathrm{x}}_{\mathrm{t}}\right)}, \overline{\mathrm{x}}(\mathrm{t})\right)\right\| \\
& \leq \mu^{*}(\mathrm{t}) \psi^{*}\left(\left\|\bar{x}_{\rho\left(\mathrm{t}, \bar{x}_{\mathrm{t}}\right)}\right\|_{\mathcal{B}}+\|\bar{x}\|\right) \\
& \leq\left\|\mu^{*}\right\|_{\mathrm{L}^{\prime}} \psi^{*}\left(\left(\mathrm{C}_{2}^{*}+\mathrm{L}^{\phi}\right)\|\phi\|_{\mathcal{B}}+\left(\mathrm{C}_{1}^{*}+1\right) \mathrm{r}\right) \\
& \leq \text { r. }
\end{aligned}
$$

Case 3. For all $t \in\left(s_{i}, t_{i+1}\right], i=1,2, \ldots, N$, we obtain

$$
\begin{aligned}
\|P(x)(t)\| & \leq\left\|Q\left(t-s_{i}\right) h_{i}\left(s_{i}, \bar{x}_{\rho\left(s_{i}, \bar{x}_{s_{i}}\right)}, \bar{x}\left(s_{i}\right)\right)\right\| \\
& +\int_{s_{i}}^{t} \int_{0}^{s}\left\|R(t-s) a(s, \tau) f\left(\tau, \bar{x}_{\rho\left(\tau, \bar{x}_{\tau}\right)}, \bar{x}(\tau)\right)\right\| d \tau d s \\
& \leq M\left\|\mu^{*}\right\|_{L^{1}} \psi^{*}\left(\left(C_{2}^{*}+L^{\phi}\right)\|\phi\|_{\mathcal{B}}+\left(C_{1}^{*}+1\right) r\right) \\
& +a C_{q, M}\|\mu\|_{L^{1}} \frac{T^{q}}{q} \psi\left(\left(C_{2}^{*}+L^{\phi}\right)\|\phi\|_{\mathcal{B}}+\left(C_{1}^{*}+1\right) r\right) \\
& \leq r .
\end{aligned}
$$

Step 3: $\mathrm{P}\left(\mathrm{B}_{\mathrm{r}}\right)$ is bounded and equicontinuous.

Case 1. For each $t \in\left[0, t_{1}\right], 0 \leq \tau_{2} \leq \tau_{1} \leq t_{1}$, and $x \in B_{r}$. Then we have

$$
\left\|\mathrm{P}(\mathrm{x})\left(\tau_{1}\right)-\mathrm{P}(\mathrm{x})\left(\tau_{2}\right)\right\| \leq \mathrm{I}_{1}+\mathrm{I}_{2}+\mathrm{I}_{3},
$$

where

$$
\begin{aligned}
& \mathrm{I}_{1}=\left\|\mathrm{Q}\left(\tau_{1}\right)-\mathrm{Q}\left(\tau_{2}\right)\right\|\|\phi(0)\| \\
& \mathrm{I}_{2}=\left\|\int_{0}^{\tau_{2}} \int_{0}^{s}\left[R\left(\tau_{1}-s\right)-R\left(\tau_{2}-s\right)\right] \mathrm{a}(s, \tau) f\left(\tau, \bar{x}_{\rho\left(\tau, \bar{x}_{\tau}\right)}, \bar{x}(\tau)\right) d \tau d s\right\| \\
& I_{3}=\left\|\int_{\tau_{2}}^{\tau_{1}} \int_{0}^{s} R\left(\tau_{1}-s\right) a(s, \tau) f\left(\tau, \bar{x}_{\rho\left(\tau, \bar{x}_{\tau}\right)}, \bar{x}(\tau)\right) d \tau d s\right\| .
\end{aligned}
$$


$I_{1}$ tends to zero as $\tau_{2} \rightarrow \tau_{1}$, since $S(t)$ is uniformly continuous operator.

For $\mathrm{I}_{2}$, using (3.2) and (H2), we have

$$
\begin{aligned}
& \mathrm{I}_{2} \leq \mathrm{a} \psi\left(\left(\mathrm{C}_{2}^{*}+\mathrm{L}^{\phi}\right)\|\phi\|_{\mathcal{B}}+\left(\mathrm{C}_{1}^{*}+1\right) \mathrm{r}\right)\|\mu\|_{\mathrm{L}^{1}} \int_{0}^{\tau_{2}}\left[\mathrm{R}\left(\tau_{1}-s\right)-\mathrm{R}\left(\tau_{2}-s\right)\right] \mathrm{d} s \\
& \leq \mathrm{a} \psi\left(\left(\mathrm{C}_{2}^{*}+\mathrm{L}^{\phi}\right)\|\phi\|_{\mathcal{B}}+\left(\mathrm{C}_{1}^{*}+1\right) \mathrm{r}\right)\|\mu\|_{\mathrm{L}^{1}} \\
& \times \int_{0}^{\tau_{2}}\left[q \int_{0}^{\infty} \sigma\left(\tau_{1}-s\right)^{q-1} \xi_{q}(\sigma) S\left(\left(\tau_{1}-s\right)^{q} \sigma\right) d \sigma\right. \\
& \left.-\mathrm{q} \int_{0}^{\infty} \sigma\left(\tau_{2}-s\right)^{\mathrm{q}-1} \xi_{\mathrm{q}}(\sigma) S\left(\left(\tau_{2}-s\right)^{\mathrm{q}} \sigma\right) \mathrm{d} \sigma\right] \mathrm{d} s \\
& \leq \mathrm{a} \psi\left(\left(\mathrm{C}_{2}^{*}+\mathrm{L}^{\phi}\right)\|\phi\|_{\mathcal{B}}+\left(\mathrm{C}_{1}^{*}+1\right) \mathrm{r}\right)\|\mu\|_{\mathrm{L}^{1}} \\
& \times\left[q \int_{0}^{\tau_{2}} \int_{0}^{\infty} \sigma \|\left[\left(\tau_{1}-s\right)^{q-1}-\left(\tau_{2}-s\right)^{q-1}\right] \xi_{q}(\sigma) S\left(\left(\tau_{1}-s\right)^{q} \sigma\right)\right. \\
& \left.+q \int_{0}^{\tau_{2}} \int_{0}^{\infty} \sigma\left(\tau_{2}-s\right)^{q-1} \xi_{q}(\sigma)\left\|S\left(\left(\tau_{1}-s\right)^{q} \sigma\right)-S\left(\left(\tau_{2}-s\right)^{q} \sigma\right)\right\|\right] \\
& \leq \mathrm{a} \psi\left(\left(\mathrm{C}_{2}^{*}+\mathrm{L}^{\phi}\right)\|\phi\|_{\mathcal{B}}+\left(\mathrm{C}_{1}^{*}+1\right) \mathrm{r}\right)\|\mu\|_{\mathrm{L}^{1}} \\
& \times \quad\left[C_{q, M} \int_{0}^{\tau_{2}}\left|\left(\tau_{1}-s\right)^{q-1}-\left(\tau_{2}-s\right)^{q-1}\right| d s\right. \\
& \left.+q \int_{0}^{\tau_{2}} \int_{0}^{\infty} \sigma\left(\tau_{2}-s\right)^{\mathrm{q}-1} \xi_{q}(\sigma)\left\|S\left(\left(\tau_{1}-s\right)^{\mathrm{q}} \sigma\right)-S\left(\left(\tau_{2}-s\right)^{\mathrm{q}} \sigma\right)\right\| \mathrm{d} \sigma d s\right] .
\end{aligned}
$$

Clearly, the first term on the right-hand side of the above inequality tends to zero as $\tau_{2} \rightarrow \tau_{1}$. From the continuity of $S(t)$ in the uniform operator topology for $t>0$, The second term on the right-hand side of the above inequality tends to zero as $\tau_{2} \rightarrow \tau_{1}$.

In view of $(\mathrm{H} 2)$, we have

$$
\begin{aligned}
I_{3} & \leq a C_{q, M} \int_{\tau_{2}}^{\tau_{1}} \int_{0}^{s}\left(\tau_{1}-s\right)^{q-1}\left\|f\left(\tau, \bar{x}_{\rho\left(\tau, \bar{x}_{\tau}\right)}, \bar{x}(\tau)\right)\right\| d \tau d s \\
& \leq a C_{q, M} \psi\left(\left(C_{2}^{*}+L^{\phi}\right)\|\phi\|_{\mathcal{B}}+\left(C_{1}^{*}+1\right) r\right)\|\mu\|_{L^{1}} \int_{\tau_{2}}^{\tau_{1}}\left(\tau_{1}-s\right)^{q-1} d s .
\end{aligned}
$$

As $\tau_{2} \rightarrow \tau_{1}, I_{3}$ tends to zero.

Case 2. For each $t \in\left[t_{i}, s_{i}\right), i=1,2, \ldots, N, t_{i} \leq \tau_{2} \leq \tau_{1} \leq s_{i}$, and $x \in B_{r}$. Then we have

$$
\begin{aligned}
\left\|P(x)\left(\tau_{1}\right)-P(x)\left(\tau_{2}\right)\right\| & =\left\|h_{i}\left(\tau_{1}, \bar{x}_{\rho\left(\tau_{1}, \bar{x}_{\tau_{1}}\right)}, \bar{x}\left(\tau_{1}\right)\right)-h_{i}\left(\tau_{2}, \bar{x}_{\rho\left(\tau_{2}, \bar{x}_{\tau_{2}}\right)}, \bar{x}\left(\tau_{2}\right)\right)\right\| \\
& \rightarrow 0 \text { as } \tau_{2} \rightarrow \tau_{1} .
\end{aligned}
$$

Case 3. For each $t \in\left(s_{i}, t_{i+1}\right], i=1,2, \ldots, N, s_{i} \leq \tau_{2} \leq \tau_{1} \leq t_{i+1}$, and $x \in B_{r}$. Then we have

$$
\left\|\mathrm{P}(\mathrm{x})\left(\tau_{1}\right)-\mathrm{P}(\mathrm{x})\left(\tau_{2}\right)\right\| \leq\left\|\mathrm{Q}\left(\tau_{1}-s_{i}\right)-\mathrm{Q}\left(\tau_{2}-s_{i}\right)\right\|\left\|h_{i}\left(s_{i}, \bar{x}_{\rho\left(s_{i}, \bar{x}_{s_{i}}\right)}, \bar{x}\left(s_{i}\right)\right)\right\|+\mathrm{I}_{1}+\mathrm{I}_{2}+\mathrm{I}_{3} .
$$

Since $S(t)$ is uniformly continuous operator, so

$$
\lim _{\tau_{2} \rightarrow \tau_{1}}\left\|Q\left(\tau_{1}-s_{i}\right)-Q\left(\tau_{2}-s_{i}\right)\right\|=0, i=1, \ldots, N .
$$


Consequently

$$
\lim _{\tau_{2} \rightarrow \tau_{1}}\left\|P(x)\left(\tau_{1}\right)-P(x)\left(\tau_{2}\right)\right\|=0
$$

Thus, $\mathrm{P}\left(\mathrm{B}_{\mathrm{r}}\right)$ is equicontinuous.

Now let $\mathrm{V}$ be a subset of $\mathrm{B}_{\mathrm{r}}$ such that $\mathrm{V} \subset \overline{\operatorname{conv}}(\mathrm{P}(\mathrm{V}) \cup\{0\})$. Moreover, for any $\varepsilon>0$ and bounded set $\mathrm{D}$, we can take a sequence $\left\{v_{n}\right\}_{n=1}^{\infty} \subset \mathrm{D}$ such that $\alpha(\mathrm{D}) \leq 2 \alpha\left(\left\{v_{n}\right\}\right)+\varepsilon([16$, P. 125). Thus, for $\left\{v_{n}\right\}_{n=1}^{\infty} \subset \mathrm{V}$, and using lemmas 2.5 2.7 and (H3), we have, for $\mathrm{t} \in\left[0, \mathrm{t}_{1}\right]$,

$$
\begin{aligned}
\alpha(P V) & \leq 2 \alpha\left(\left\{P v_{n}\right\}\right)+\varepsilon \\
& =2 \sup _{t \in J} \alpha\left(\left\{P v_{n}(t)\right\}\right)+\varepsilon \\
& =2 \sup _{t \in J} \alpha\left(\left\{\int_{0}^{t} R(t-s) \int_{0}^{s} a(s, \tau) f\left(\tau, y_{\tau}+v_{n \tau}, y(\tau)+v_{n}(\tau)\right) d \tau d s\right\}\right)+\varepsilon \\
& \leq 4 \sup _{t \in J} \int_{0}^{t} \alpha\left(\left\{R(t-s) \int_{0}^{s} a(s, \tau) f\left(\tau, y_{\tau}+v_{n \tau}, y(\tau)+v_{n}(\tau)\right) d \tau d s\right\}\right)+\varepsilon \\
& \leq 8 \sup _{t \in J} \int_{0}^{t} \int_{0}^{s} \alpha\left(\left\{R(t-s) a(s, \tau) f\left(\tau, y_{\tau}+v_{n \tau}, y(\tau)+v_{n}(\tau)\right) d \tau d s\right\}\right)+\varepsilon \\
& \leq 8 a \sup _{t \in J} \int_{0}^{t} \int_{0}^{s} \alpha\left(\left\{R(t-s) f\left(\tau, y_{\tau}+v_{n \tau}, y(\tau)+v_{n}(\tau)\right) d \tau d s\right\}\right)+\varepsilon \\
& \leq 8 a \sup _{t \in J} \int_{0}^{t} \int_{0}^{s} \eta_{t}(s, \tau)\left[\alpha\left(v_{n}(\tau)\right)+\sup _{-\infty<\theta \leq 0} \alpha\left(v_{n}(\theta+\tau)\right)\right] d \tau d s+\varepsilon \\
& \leq 8 a \sup _{t \in J}^{t} \int_{0}^{t} \int_{0}^{s} \eta_{t}(s, \tau)\left[\alpha\left(v_{n}\right)+\sup _{0<\mu \leq \tau} \alpha\left(v_{n}(\mu)\right)\right] d \tau d s+\varepsilon \\
& \leq 16 a \alpha\left(v_{n}\right) \sup _{t \in J} \int_{0}^{t} \int_{0}^{s} \eta_{t}(s, \tau) d \tau d s+\varepsilon \\
& \leq 16 a \eta^{*} \alpha(V)+\varepsilon .
\end{aligned}
$$

For any $t \in\left[t_{i}, s_{i}\right), i=1,2, \ldots, N$, we get

$$
\begin{aligned}
\alpha(P V) & =\alpha\left(h_{i}\left(t, \bar{x}_{\rho\left(t, \bar{x}_{t}\right)}, \bar{x}(t)\right)\right) \\
& \leq \mathrm{L}\left(\alpha\left(v_{n}(t)\right)+\sup _{-\infty<\theta \leq 0} \alpha\left(v_{n}(\theta+t)\right)\right) \\
& \leq \mathrm{L}\left(\alpha\left(v_{n}\right)+\sup _{0<\mu \leq \tau} \alpha\left(v_{n}(\mu)\right)\right) \\
& \leq 2 \mathrm{~L} \alpha\left(v_{n}\right) \\
& \leq 2 \mathrm{~L} \alpha(V) .
\end{aligned}
$$


In the same way, for any $t \in\left(s_{i}, t_{i+1}\right], i=1,2, \ldots, N$, we obtain

$$
\begin{aligned}
\alpha(P V) & \leq 2 \alpha\left(\left\{P v_{n}\right\}\right)+\varepsilon \\
& =2 \sup _{t \in J} \alpha\left(\left\{P v_{n}(t)\right\}\right)+\varepsilon \\
& =2 \sup _{t \in J} \alpha\left(Q\left(t-s_{i}\right) h_{i}\left(s_{i}, \bar{x}_{\rho\left(s_{i}, \bar{x}_{s_{i}}\right)}, \bar{x}\left(s_{i}\right)\right)\right) \\
& +2 \sup _{t \in J} \alpha\left(\left\{\int_{s_{i}}^{t} R(t-s) \int_{0}^{s} a(s, \tau) f\left(\tau, y_{\tau}+v_{n \tau}, y(\tau)+v_{n}(\tau)\right) d \tau d s\right\}\right)+\varepsilon \\
& \leq 2 M L \alpha\left(v_{n}\right) \\
& +4 \sup _{t \in J}^{t} \int_{s_{i}}^{t} \alpha\left(\left\{R(t-s) \int_{0}^{s} a(s, \tau) f\left(\tau, y_{\tau}+v_{n \tau}, y(\tau)+v_{n}(\tau)\right) d \tau d s\right\}\right)+\varepsilon \\
& \leq 2 M L \alpha\left(v_{n}\right) \\
& +8 \sup _{t \in J} \int_{s_{i}}^{t} \int_{0}^{s} \alpha\left(\left\{R(t-s) a(s, \tau) f\left(\tau, y_{\tau}+v_{n \tau}, y(\tau)+v_{n}(\tau)\right) d \tau d s\right\}\right)+\varepsilon \\
& \leq 2 M L \alpha\left(v_{n}\right)+8 a \sup _{t \in J} \int_{s_{i}}^{t} \int_{0}^{s} \alpha\left(\left\{R(t-s) f\left(\tau, y_{\tau}+v_{n \tau}, y(\tau)+v_{n}(\tau)\right) d \tau d s\right\}\right)+\varepsilon \\
& \leq 2 M L \alpha\left(v_{n}\right)+8 a \sup _{t \in J} \int_{s_{i}}^{t} \int_{0}^{s} \eta_{t}(s, \tau)\left[\alpha\left(v_{n}(\tau)\right)+\sup _{-\infty<\theta \leq 0} \alpha\left(v_{n}(\theta+\tau)\right)\right] d \tau d s+\varepsilon \\
& \leq 2 M L \alpha\left(v_{n}\right)+8 a \sup _{t \in J} \int_{s_{i}}^{t} \int_{0}^{s} \eta_{t}(s, \tau)\left[\alpha\left(v_{n}\right)+\sup _{0<\mu \leq \tau} \alpha\left(v_{n}(\mu)\right)\right] d \tau d s+\varepsilon \\
\leq & 2 M L \alpha\left(v_{n}\right)+16 a \alpha\left(v_{n}\right) \sup _{t \in J} \int_{0}^{t} \int_{0}^{s} \eta_{t}(s, \tau) d \tau d s+\varepsilon \\
\leq & 2 M L \alpha(V)+16 a \eta^{*} \alpha(V)+\varepsilon \\
\leq & \left(2 M L+16 a \eta^{*}\right) \alpha(V)+\varepsilon .
\end{aligned}
$$

Therefore, in view of Lemma 2.5, we have

$$
\alpha(\mathrm{V}) \leq \alpha(\mathrm{PV}) \leq\left(2 \mathrm{ML}+16 \mathrm{a} \eta^{*}\right) \alpha(\mathrm{V})+\varepsilon,
$$

since $\varepsilon$ is arbitrary we obtain that

$$
\alpha(V) \leq\left(2 M L+16 a \eta^{*}\right) \alpha(V)
$$

This means that

$$
\alpha(V)\left(1-\left(2 M L+16 a \eta^{*}\right)\right) \leq 0 .
$$

By (3.3) it follows that $\alpha(\mathrm{V})=0$. In view of the Ascoli-Arzelà theorem, $\mathrm{V}$ is relatively compact in $\mathrm{B}_{\mathrm{r}}$. Applying now Theorem 2.10, we conclude that $\mathrm{P}$ has a fixed point which is a solution of the problem (1.1).

The second result is established using the Darbo's fixed point theorem. 
Theorem 3.8. Assume that $(\mathrm{H} 1)-(\mathrm{H} 5)$ are satisfied, then the problem (1.1) has at least one mild solution.

Proof. In what follows we show that the operator $P: Y \rightarrow Y$ is a strict set contraction. We know that $\mathrm{P}: \mathrm{Y} \rightarrow \mathrm{Y}$ is bounded and continuous, we need to prove that there exists a constant $0 \leq v<1$ such that $\alpha(P V) \leq v \alpha(V)$ for $V \subset B_{r}$.

Using the same method as the proof of Theorem 3.7, for $t \in[0, T]$, we have

$$
\alpha(P V) \leq\left(2 M L+16 a \eta^{*}\right) \alpha(V)+\varepsilon
$$

since $\varepsilon$ is arbitrary we obtain that

$$
\alpha(P V) \leq v \alpha(V)
$$

Hence $\mathrm{P}$ is a set contraction. According to Theorem 2.11 the operator $\mathrm{P}$ has at least one fixed point which is obviously a mild solution of the problem (2.4). This completes the proof.

\section{An Example}

We consider the fractional integro-differential equations with state-dependent delay and noninstantaneous impulses of the form

$$
\begin{aligned}
& \frac{\partial_{t}^{q}}{\partial t^{q}} v(t, \zeta)+\frac{\partial^{2}}{\partial \zeta^{2}} v(t, \zeta)=\int_{0}^{t}(t-s)^{2} \int_{-\infty}^{s} \gamma(\tau-s) v\left(\tau-\rho_{1}(s) \rho_{2}(|v(s, \zeta)|), \zeta\right) d \tau d s \\
& +\int_{0}^{t}(t-s)^{2} \cos |v(s, \zeta)| d s,(t, x) \in \mathbb{N} \in \cup_{i=1}^{n}\left[s_{i}, t_{i+1}\right] \times[0, \pi], \\
& v(t, 0)=v(t, \pi)=0, \quad t \in[0, T], \\
& v(\tau, \zeta)=v_{0}(\theta, \zeta), \quad \theta \in(-\infty, 0], x \in[0, \pi] \\
& v(t, \zeta)=H_{i}\left(t, v\left(t-\rho_{1}(t) \rho_{2}(|v(t, \zeta)|), \zeta\right), \zeta\right),(t, x) \in\left(t_{i}, s_{i}\right] \times[0, \pi], i=1,2, \ldots, N,
\end{aligned}
$$

where $0<\mathrm{q}<1,0=\mathrm{t}_{0}=\mathrm{s}_{0}<\mathrm{t}_{1} \leq \mathrm{s}_{1} \leq \mathrm{t}_{2}<\ldots<\mathrm{t}_{\mathrm{N}-1} \leq \mathrm{s}_{\mathrm{N}} \leq \mathrm{t}_{\mathrm{N}} \leq \mathrm{t}_{\mathrm{N}+1}=\mathrm{T}$ are prefixed real numbers and the functions $\gamma: \mathbb{R} \rightarrow \mathbb{R}, \rho_{i}:[0,+\infty) \rightarrow[0,+\infty), i=1,2$ are continuous functions.

Let $X=L^{2}([0, \pi])$ and define the operator $A: D(A) \subset X \rightarrow X$ by $A \omega=\omega^{\prime \prime}$ with domain

$$
D(A)=\left\{\omega \in E: \omega, \omega^{\prime} \text { are absolutely continuous, } \omega^{\prime \prime} \in E, \omega(0)=\omega(\pi)=0\right\} .
$$

Then

$$
A \omega=\sum_{n=1}^{\infty} n^{2}\left(\omega, \omega_{n}\right) \omega_{n}, \quad \omega \in D(A),
$$


where $\omega_{n}(x)=\sqrt{\frac{2}{\pi}} \sin (n x), n \in \mathbb{N}$ is the orthogonal set of eigenvectors of $A$. It is well known that $A$ is the infinitesimal generator of an analytic semigroup $\{S(t)\}_{t} \geq 0$ in $X$ and is given by

$$
\mathrm{S}(\mathrm{t}) \omega=\sum_{n=1}^{\infty} e^{-n^{2} \mathrm{t}}\left(\omega, \omega_{n}\right) \omega_{n}, \quad \forall \omega \in X, \text { and every } \mathrm{t}>0
$$

From these expressions, it follows that $\{\mathrm{S}(\mathrm{t})\}_{\mathrm{t} \geq 0}$ is a uniformly bounded compact semigroup on $\mathrm{X}$. For the phase space, we choose $\mathcal{B}=\mathrm{C}_{0} \times \mathrm{L}^{2}(\mathrm{~g}, \mathrm{X})$, see Example 2.9 for details.

Set

$$
\begin{aligned}
x(t)(\zeta) & =v(t, \zeta), \\
\phi(\theta)(\zeta) & =v_{0}(\theta, \zeta), \\
a(t, s) & =(t-s)^{2} \\
f(t, \varphi, x(t))(\zeta) & =\int_{-\infty}^{0} \gamma(t) \varphi(t, \zeta) d s+\cos |x(t)(\zeta)|, \\
h_{i}(t, \varphi, x(t))(\zeta) & =H_{i}\left(t, v\left(t-\rho_{1}(t) \rho_{2}(|x(t)|), \zeta\right), \zeta\right) \\
\rho(t, \varphi) & =t-\rho_{1}(t) \rho_{2}(|\varphi(0)|) .
\end{aligned}
$$

Under the above conditions, we can represent the problem (4.1) by the abstract problem (1.1).

Proposition 4.1. Let $\varphi \in \mathcal{B}$ be such that $\left(\mathrm{H}_{\varphi}\right)$ holds, and let $\mathrm{t} \rightarrow \varphi_{\mathrm{t}}$ be continuous on $\mathcal{R}\left(\rho^{-}\right)$. Then there exists a mild solution of (4.1). 


\section{References}

[1] S. Abbas, M. Benchohra, J. Graef and J. Henderson, Implicit Fractional Differential and Integral Equations; Existence and Stability, De Gruyter, Berlin, 2018.

[2] S. Abbas, M. Benchohra and G.M. N'Guérékata, Topics in Fractional Differential Equations, Springer, New York, 2012.

[3] S. Abbas, M. Benchohra and G.M. N'Guérékata, Advanced Fractional Differential and Integral Equations, Nova Science Publishers, New York, 2015.

[4] R. P. Agarwal, S. Hristova, and D. O'Regan, Non-instantaneous Impulses in Differential Equations. Springer, Cham, 2017.

[5] R. P. Agarwal, M. Meehan, and D. O'Regan, Fixed Point Theory and Applications, Cambridge University Press, Cambridge, 2001.

[6] R.P. Agarwal, M. Benchohra and B.A. Slimani, Existence results for differential equations with fractional order impulses, Mem. Differential Equations. Math. Phys., 44 (2008), 1-21.

[7] A. Anguraj and P. Karthikeyan, Anti-periodic boundary value problem for impulsive fractional integro differential equations, Fract. Calc. Appl. Anal. 13 (2010), 1-13.

[8] A. Anguraj and S. Kanjanadevi, Existence results for fractional non-instantaneous impulsive integro-differential equations with nonlocal conditions, Dynam. Cont. Disc. Ser. A 23 (2016), 429-445.

[9] A. Anguraj and S. Kanjanadevi, Non-instantaneous impulsive fractional neutral differential equations with state-dependent delay, Progr. Fract. Differ. Appl. 3(3) (2017), 207-218.

[10] K. Balachandran and S. Kiruthika, Existence of solutions of abstract fractional impulsive semilinear evolution equations, Electron. J. Qual. Theor. Differ. Equat., 2010(4)(2010), 1-12.

[11] K. Balachandran, S.Kiruthika and J.J. Trujillo, Existence results for fractional impulsive integrodifferential equations in Banach spaces, Commun. Nonlinear Sci. Num. Simul. 16 (2011), 1970-1977.

[12] D. Baleanu, K. Diethelm, E. Scalas, J.J. Trujillo, Fractional Calculus Models and Numerical Methods, World Scientific Publishing, New York, 2012.

[13] J. Banaś and K. Goebel, Measures of Noncompactness in Banach Spaces, of Lecture Notes in Pure and Applied Mathematics, Marcel Dekker, New York, 1980.

[14] M. Benchohra, J. Henderson and S. K. Ntouyas, Impulsive Differential Equations and Inclusions, Hindawi Publishing Corporation, Vol 2, New York, 2006. 
[15] M. Benchohra and S. Litimein, Existence results for a new class of fractional integro-differential equations with state dependent delay, Mem. Differ. Equa. Math. Phys. 74 (2018), 27-38.

[16] D. Bothe, Multivalued perturbations of m-accretive differential inclusions, Israel J. Math. 108 (1998), 109-138.

[17] L. Debnath and D. Bhatta, Integral Transforms and Their Applications (Second Edition), CRC Press, 2007.

[18] K. Diethelm, The Analysis of Fractional Differential Equations. Springer, Berlin, 2010.

[19] G. R. Gautam and J. Dabas, Existence result of fractional functional integro-differential equation with not instantaneous impulse, Int. J. Adv. Appl. Math. Mech. 1(3) (2014), 11-21.

[20] J. K. Hale and J. Kato, Phase space for retarded equations with infinite delay, Funk. Ekvacioj, 21 (1) (1978), 11-41.

[21] H. P. Heinz, On the behaviour of measures of noncompactness with respect to differentiation and integration of vector-valued functions, Nonlinear Anal. 7 (12) (1983), 1351-1371.

[22] E. Hernández, A. Prokopczyk, and L. Ladeira, A note on partial functional differential equations with state-dependent delay, Nonlinear Anal. RWA, 7 (2006), 510-519.

[23] E. Hernández and D. O'Regan, On a new class of abstract impulsive differential equations, Proc. Amer. Math. Soc. 141 (2013), 1641-1649

[24] R. Hilfer, Applications of Fractional Calculus in Physics. Singapore, World Scientific, 2000.

[25] Y. Hino, S. Murakami, and T. Naito, Functional Differential Equations with Unbounded Delay, Springer-Verlag, Berlin, 1991.

[26] A. A. Kilbas, Hari M. Srivastava, and Juan J. Trujillo, Theory and Applications of Fractional Differential Equations. Elsevier Science B.V., Amsterdam, 2006.

[27] P. Kumar, R. Haloi, D. Bahuguna and D. N. Pandey, Existence of solutions to a new class of abstract non-instantaneous impulsive fractional integro-differential equations, Nonlin. Dynam. Syst. Theor. 16 (1) (2016), 73-85.

[28] V. Lakshmikantham, D.D. Bainov and P.S. Simeonov, Theory of Impulsive Differential Equations, World Scientific, NJ, 1989.

[29] P. Li and C. J. Xu , Mild solution of fractional order differential equations with not instantaneous impulses, Open Math, 13 (2015), 436-443. 
[30] F. Mainardi, P. Paradisi and R. Gorenflo, Probability distributions generated by fractional diffusion equations, in Econophysics: An Emerging Science, J. Kertesz and I. Kondor, Eds., Kluwer Academic Publishers, Dordrecht, The Netherlands, 2000.

[31] M. Meghnafi, M. Benchohra and K. Aissani, Impulsive fractional evolution equations with state-dependent delay, Nonlinear Stud. 22 (4)(2015), 659-671.

[32] K. S. Miller and B. Ross, An Introduction to the Fractional Calculus and Differential Equations, John Wiley, New York, 1993.

[33] H. Mönch, Boundary value problems for nonlinear ordinary differential equations of second order in Banach spaces. Nonlinear Anal. 4 (1980), 985-999.

[34] D. N. Pandey, S. Das and N. Sukavanam, Existence of solution for a second-order neutral differential equation with state dependent delay and non-instantaneous impulses, Int. J. Nonlin. Sci. 18(2)(2014), 145-155.

[35] M. Pierri, D. O'Regan and V. Rolnik, Existence of solutions for semi-linear abstract differential equations with not instantaneous impulses, Appl. Math. Comput. 219 (2013), 6743- 6749.

[36] I. Podlubny, Fractional Differential Equations, Academic Press, San Diego, 1999.

[37] S. G. Samko, A. A. Kilbas and O. I. Marichev, Fractional Integrals and Derivatives. Theory and Applications, Gordon and Breach, Yverdon, 1993.

[38] V. E. Tarasov, Fractional Dynamics: Application of Fractional Calculus to Dynamics of Particles, Fields and Media, Springer, Heidelberg; Higher Education Press, Beijing, 2010.

[39] Y. Zhou, Fractional Evolution Equations and Inclusions: Analysis and Control, Academic Press Elsevier, 2016. 